\title{
Association between Glycemic Control and Clinic Attendance in Emerging Adults with Type 1 Diabetes: A Tertiary Center Experience
}

\author{
Eldad Fisher, ${ }^{1}$ Liora Lazar, ${ }^{1,2}$ Shlomit Shalitin, ${ }^{1,2}$ Michal Yackobovitch-Gavan, ${ }^{2}$ \\ Liat de Vries, ${ }^{1,2}$ Tal Oron, ${ }^{2}$ Ariel Tenenbaum, ${ }^{1,2}$ Moshe Phillip, ${ }^{1,2}$ and Yael Lebenthal ${ }^{10,2}$ \\ ${ }^{1}$ Sackler Faculty of Medicine, Tel Aviv University, Tel Aviv 69978, Israel \\ ${ }^{2}$ The Jesse Z and Sara Lea Shafer Institute for Endocrinology and Diabetes, National Center for Childhood Diabetes, \\ Schneider Children's Medical Center of Israel, Petah Tikva 49202, Israel
}

Correspondence should be addressed to Yael Lebenthal; yael.lebenthal@gmail.com

Received 13 March 2018; Revised 21 May 2018; Accepted 19 June 2018; Published 9 July 2018

Academic Editor: Andrea Scaramuzza

Copyright (C) 2018 Eldad Fisher et al. This is an open access article distributed under the Creative Commons Attribution License, which permits unrestricted use, distribution, and reproduction in any medium, provided the original work is properly cited.

\begin{abstract}
Aims. The transition of emerging adults with type 1 diabetes (T1D) from pediatric diabetes clinics to adult clinics between 18 and 21 years of age could result in decreased clinic attendance and thus worsen glycemic control. Our institutional policy offering surveillance till age 30 enabled us to evaluate clinic attendance without the confounding effect of transition. Our aim was to determine the association between glycemic control (HbAlc) and attendance rate. Methods. The medical records of 261 (54\% males) young adult T1D patients (median age 22.9 years) were reviewed. Patients were stratified according to the attainment/ nonattainment of glycemic targets $(\mathrm{HbAlc} \leq 7 \%$ versus $\mathrm{HbAlc}>7 \%(53 \mathrm{mmol} / \mathrm{mol}))$. The attendance rate was calculated as the number of clinic visits/number of scheduled appointments. Results. Median annual number of scheduled visits was 3 (3, 4); attendance rate was $75 \%(53.6 \%, 100 \%)$. Seventy-four $(28.4 \%)$ patients attained glycemic targets (median $\mathrm{HbA1c} 6.5 \%$ $(48 \mathrm{mmol} / \mathrm{mol})(6.3 \%, 6.8 \%(45.51 \mathrm{mmol} / \mathrm{mol})) ; 187(71.6 \%)$ patients had a median $\mathrm{HbAlc}$ of $7.8 \%(62 \mathrm{mmol} / \mathrm{mol})(7.4 \%, 8.4 \%$ $(57.68 \mathrm{mmol} / \mathrm{mol}))$. The attainment of the treatment target was more prevalent in older patients $(P=0.006)$, in male patients $(P=0.007)$, and in patients with higher education $(P=0.017)$. Higher attendance rate $(\beta(2.483), P<0.001)$ and male gender ( $\beta$ (0.746), $P=0.015)$ were associated with better metabolic control. Conclusions. In emerging adults with T1D during the ongoing stable phase of diabetes management, higher attendance rate, rather than absolute number of clinic visits, was associated with the attainment of glycemic targets.
\end{abstract}

\section{Introduction}

The Diabetes Control and Complications Trial (DCCT), based on a regimen of monthly clinic visits with a multidisciplinary team, clearly demonstrated the positive impact of intensive diabetes therapy on glycemic control [1]. Since then, it has come to be widely accepted that the more practical regimen of 3-4 annual visits, as advocated by the American Diabetes Association (ADA), constitutes a satisfactory therapeutic regimen for adults with $\operatorname{T1D}[2,3]$. The routine clinical practice followed for young adults in our tertiary center for diabetes has been quarterly clinical visits during which $\mathrm{HbAlc}$ is evaluated and diabetes management is reassessed. Yet, diabetologists can recommend a fewer or greater number of visits per their clinical judgement [3]. T1D patients who have unsatisfactory glycemic control are invited more frequently in an attempt to promote adherence to treatment regimen.

Emerging adulthood (defined as age $18-30$ years) is a period when patients are expected to assume greater responsibility for their diabetes care, while parents reduce their involvement in management of the disease [4]. Current guidelines endorse the transition of T1D patients from pediatric diabetes clinics to adult clinics between 18 and 21 years 
of age [5]. Several groups have reported concern that the transition of emerging adults at this young age could result in decreased clinic attendance and thus worsening glycemic control [5-9]. Our institutional policy in the past two decades has been to offer continuing surveillance by our multidisciplinary team until the much later age of 30 years. This practice has enabled us to determine whether clinic attendance affects glycemic control in emerging adults with T1D without the confounding effect of transition. Our hypothesis was that well-controlled type 1 diabetes-emerging adult patients could attend the clinic less frequently than 4 times a year. The objective of the present study was to determine the association between glycemic control ( $\mathrm{HbAlc}$ ) and clinic attendance rate during the period of emerging adulthood and to assess the influence of sociodemographic and diabetesrelated parameters on clinic attendance.

\section{Patients and Methods}

2.1. Patients. A survey of all patients attending the National Center for Childhood Diabetes at the Schneider Children's Medical Center of Israel (SCMCI) between January 1st, 2011 and December 31st, 2011, yielded 397 emerging adult T1D patients aged 18 to 30 years. Of these, 261 patients fulfilled the following inclusion criteria: duration of T1D for at least one year prior to data retrieval with follow-up during 2012 (indicating continued surveillance). Excluded from the study were 136 patients: 45 discontinued follow-up in our center, 33 required more intensive diabetes surveillance, with $>4$ clinic visits, 21 participated in an intervention study, 21 changed mode of therapy from multiple daily injections (MDI) to continuous subcutaneous insulin infusion (CSII) and participated in a 3-day education session, and 16 had a recent onset of diabetes. During the studied period of surveillance, the population of emerging adults was categorized as follows: $65.7 \%$ (261/397) - ongoing stable phase of diabetes management (defined as the period beyond initial diagnosis and early diabetes education-the study cohort); $23 \%$ (91/ 397)-more intensive diabetes management (recent onset of T1D, more frequent clinic visits due to participation in an intervention study or changing mode of therapy); and $11.3 \%$ (45/397) - discontinued follow-up in our center.

The study protocol was approved by our Institutional Review Board. Informed consent was waivered by our Institutional Review Board.

2.2. Methods. This was a retrospective study of a cohort of emerging adult T1D patients followed at our tertiary center for diabetes. Retrieved from medical files were sociodemographic parameters (age, gender, ethnicity, region of residence, distance from clinic, and employment status) and diabetes-related parameters (age at diagnosis of T1D, number of clinic visits, number of appointments made, $\mathrm{HbAlc}$ levels, mode of therapy, whether MDI or CSII, episodes of DKA, severe hypoglycemia, and diabetes-related hospitalizations).

Attendance (kept appointment) rate was calculated as the number of clinic visits/number of scheduled appointments. Capillary HbA1c was measured by an automated immunochemical technique (DCA 2000; Bayer Diagnostics Inc.,
Tarrytown, NY, USA; 95\% confidence interval (CI) 4.3$5.7 \%)$. HbAlc was routinely tested at each visit. Median $\mathrm{HbA1c}$ was calculated for each patient using the available $\mathrm{HbAlc}$ values during the one-year study period. Target $\mathrm{HbAlc}$ was defined according to ADA guidelines in emerging adults as $\leq 7 \%(53 \mathrm{mmol} / \mathrm{mol})$ [10]. DKA was defined as an episode of hyperglycemia and ketoacidosis leading to an emergency department visit and/or hospital admission. Severe hypoglycemia was defined as a hypoglycemic episode associated with severe cognitive impairment requiring external assistance for recovery and treated at home with glucagon, severe cognitive impairment requiring the assistance of another person or requiring assistance in an emergency department visit, or severe cognitive impairment requiring hospital admission. Data on DKA and/or severe hypoglycemia occurring during the preceding months were reported and recorded at each visit.

2.3. Statistical Analysis. The data were analyzed using SPSS statistical software (release 23.0; SPSS Inc., Chicago, IL). All statistical tests were performed as two sided. The Kolmogorov-Smirnov $z$ test was performed to test the null hypothesis that the variable has a normal distribution. Data are expressed as median and interquartile range (IQR) for skewed distribution. HbAlc was stratified into 2 categories $(\leq 7 \%(53 \mathrm{mmol} / \mathrm{mol}),>7 \%(53 \mathrm{mmol} / \mathrm{mol}))$ and comparisons of demographic and clinical parameters were performed. Pearson's chi-square test was used for analysis of betweengroup differences in discrete variables. Independent-samples Mann-Whitney $U$ test was used to compare between groups for continuous variables (all had a skewed distribution). Stepwise multivariate logistic regression analysis (backward LR) was used to define factors potentially affecting target $\mathrm{HbAlc}$. A $P$ value of $\leq 0.05$ was considered significant.

\section{Results}

The cohort of 261 emerging adults (54.4\% males) had a median age of 22.9 years (IQR 20.7, 25.9). Median age at diagnosis of T1D was 11.8 (IQR 10.4, 12.9) with a median diabetes duration of 11.1 years (IQR 7.8, 15.5). Sociodemographic characteristics of the entire cohort revealed that only a minority were unemployed (3.8\%), and only a few (1.1\%) did not graduate high school. Most resided in an urban area (78.9\%), with a median distance from the clinic of $20 \mathrm{~km}$ (IQR 10, 41).

In the entire cohort, the median number of annual scheduled visits was 3 (IQR 3,4) and the attendance rate was $75 \%$ (IQR $53.6 \%, 100 \%$ ). During this period, treatment modality was MDI in $58.6 \%$ of the emerging adults and CSII in 41.4\%. Autoimmune comorbidities were found in $13.2 \%$ of the cohort and microvascular complications in $7.3 \%$. Acute diabetes-related complications-severe hypoglycemic events, DKA episodes, and diabetes-related hospitalizations-were documented in $1.5 \%, 1.1 \%$, and $2.3 \%$ of the patients, respectively.

Median HbAlc of the studied cohort was 7.5\% (59 mmol/mol) (IQR $(6.9 \%, 8.1 \%) \quad(52,65 \mathrm{mmol} / \mathrm{mol})$ ). Seventy-four $(28.4 \%)$ of the patients attained treatment 
TABLE 1: Sociodemographic characteristics of emerging adults with type 1 diabetes stratified by the attainment of treatment targets.

\begin{tabular}{|c|c|c|c|}
\hline $\mathrm{HbAlc}$ \% (mmol/mol) & $\leq 7(53)$ & $>7(53)$ & $P$ \\
\hline Patients, $n(\%)$ & $74(28.4)$ & $187(71.6)$ & \\
\hline Current age, median (IQR), years & $23.9(21.4,27.1)$ & $22.5(20.5,25.2)$ & 0.006 \\
\hline \multicolumn{4}{|l|}{ Gender, n (\%) } \\
\hline Male gender & $50 / 142(35.2)$ & $92 / 142(64.8)$ & \multirow{2}{*}{0.007} \\
\hline Female gender & $24 / 119(20.2)$ & $95 / 119(79.8)$ & \\
\hline \multicolumn{4}{|l|}{ Ethnicity, $n(\%)$} \\
\hline Jewish & $71 / 245(29.0)$ & $174 / 245(71.0)$ & \multirow{2}{*}{0.191} \\
\hline Arab & $2 / 15(13.3)$ & $13 / 15(86.7)$ & \\
\hline \multicolumn{4}{|l|}{ Employment, $n(\%)$} \\
\hline Unemployed & $1(1.4)$ & $9(4.8)$ & \multirow{4}{*}{0.129} \\
\hline Military/national service & $8(10.8)$ & $34(18.2)$ & \\
\hline Student & $30(40.5)$ & $79(42.2)$ & \\
\hline Employed & $35(47.3)$ & $65(34.8)$ & \\
\hline \multicolumn{4}{|l|}{ Adult education, $n$ (\%) } \\
\hline Elementary & $1(1.4)$ & $2(1.1)$ & \multirow{3}{*}{0.017} \\
\hline High school & $49(66.2)$ & $154(82.8)$ & \\
\hline University & $24(32.4)$ & $31(16.6)$ & \\
\hline Distance from clinic, median (IQR), $\mathrm{km}$ & $15.5(8,37)$ & $20(10,41)$ & 0.132 \\
\hline
\end{tabular}

targets (HbAlc $\leq 7 \%)$ with a median $\mathrm{HbA} 1 \mathrm{c}$ of $6.5 \%$ $(48 \mathrm{mmol} / \mathrm{mol})(\mathrm{IQR}(6.3 \%, 6.8 \%)(45,51 \mathrm{mmol} / \mathrm{mol})) ; 187$ (71.6\%) of the patients had a median $\mathrm{HbA1c}$ of $7.8 \%$ $(62 \mathrm{mmol} / \mathrm{mol})$ (IQR $(7.4 \%, 8.4 \%)(57,68 \mathrm{mmol} / \mathrm{mol}))$. Patients who attained treatment targets had a significantly lower number of scheduled visits (median (IQR): $2(2,3)$ versus $3(2,3), P<0.001)$ but a higher attendance rate (median (IQR): $100(67,100)$ versus $75(50,100), P=0.020)$.

Sociodemographic characteristics (Table 1) showed that the attainment of the treatment target was more prevalent in older patients $(P=0.006)$, in male patients $(P=$ $0.007)$, and in patients with higher education $(P=0.017)$. Ethnicity, employment status, and distance from the diabetes clinic did not significantly affect the attainment of glycemic control.

Diabetes-related characteristics of the studied cohort according to the attainment of treatment targets is presented in Table 2. Median age at diagnosis of diabetes was significantly older in patients attaining treatment targets (13.3 versus 11.5 years, $P=0.020)$, with no significant difference in diabetes duration and autoimmune comorbidities. Treatment modality was similar among patients with $\mathrm{HbA} 1 \mathrm{c} \leq 7 \%$ and those with $>7 \%$ (CSII in $44.6 \%$ versus $40.1 \%, P=0.600)$. No significant differences were found between groups in regard to diabetes-related complications per 100 patient-years (hypoglycemic events: 2.7 versus 1.1, $P=0.333$, diabetic ketoacidosis: 1.4 versus $1.1, P=0.847$, diabetes-related hospitalizations: 2.7 versus $2.2, P=0.789$, and microvascular complications: 0.8 versus $0.6, P=0.454$ ).

Predictors for metabolic control in emerging adults are presented in Table 3. The final logistic regression model showed that a better kept attendance rate $(\beta$ (2.483), $P<0.001)$ and male gender $(\beta$ (0.746), $P=0.015)$ were associated with a better metabolic control. This model explains $11.4 \%$ of the variance in HbA1c levels $\left(R^{2}=0.114\right)$.

\section{Discussion}

Our institutional policy offering continuing surveillance of young adults up to age 30 within the framework of our pediatric services enabled us to evaluate the importance of clinic attendance as recommended by the multidisciplinary diabetes team without the confounding effect of transition. The present study revealed that in emerging adult patients with T1D during the ongoing stable phase of diabetes management, a higher kept appointment rate was associated with the attainment of better glycemic control.

During the period of emerging adulthood, T1D patients are at increased risk of a declining frequency of clinic visits and even possible disengagement from health care surveillance $[6-8,11,12]$. Although our institutional policy encourages all emerging adults to remain under our surveillance, $11 \%$ of the patients in this cohort discontinued their followup in our center. Discontinuation of follow-up in our institute cannot be attributed to the financial burden of healthcare since in Israel healthcare service is mandated for all residents via the endorsement of the Health Insurance Law. Diabetes patients benefit from comprehensive, publicly funded, and administered care which includes doctor visits, diagnostic and laboratory services, hospitalization, and subsidized prescription medications.

Our emerging adult cohort who continued their followup in our clinic attended an average of $75 \%$ of scheduled visits, an outcome better than previously reported by others [11-13]. This relatively high kept appointment rate may stem from an ascertainment bias as motivated young adults are 
TABLE 2: Comparison of diabetes-related characteristics of emerging adults with type 1 diabetes during the studied year stratified by the attainment of treatment targets.

\begin{tabular}{lcc}
\hline HbAlc, \% (mmol/mol) & $\leq 7(53)$ & $>7(53)$ \\
\hline Patients, $n$ (\%) & 74 & 187 \\
Age at diagnosis, median (IQR), years & $13.3(10.1,16.3)$ & $11.5(8.8,14.4)$ \\
Duration of diabetes, median (IQR), years & $11.4(7.1,16.1)$ & $11.0(8.2,15.3)$ \\
Autoimmune comorbidities, $n$ (\%) & $13(17.6)$ & $33(17.6)$ \\
Current treatment modality, $n$ (\%) & $41(55.4)$ & 0.020 \\
$\quad$ Multiple daily injections & $33(44.6)$ & $112(59.9)$ \\
$\quad$ Insulin pump & $3(2,4)$ & $75(40.1)$ \\
Annual number of scheduled visits, median (IQR) & $2(2,3)$ & $4(3,5)$ \\
Annual number of clinic visits, median (IQR) & $100(67,100)$ & $3(2,3)$ \\
Attendance rate, median (IQR), percentage & 2.7 & $75(50,100)$ \\
Presence of hypoglycemic events, per 100 patient-years & 1.4 & 0.600 \\
Presence of diabetic ketoacidosis, per 100 patient-years & 2.7 & 0.043 \\
Presence of diabetes-related hospitalizations, per 100 patient-years & 0.83 & 0.001 \\
Microvascular complications, per 100 patient-years & 0.333 \\
\hline
\end{tabular}

Data are presented as median and interquartile range (IQR) unless otherwise indicated. HbAlc, glycated hemoglobin; $n$, number; $\%$, percentage. $P \leq 0.05$ was considered significant.

TABLE 3: Final logistic regression model for variables associated with the attainment of target $\mathrm{HbAlc}$.

\begin{tabular}{lcccc}
\hline & $\beta(\mathrm{SE})$ & $P$ & $\mathrm{OR}$ & $95 \%$ CI for OR \\
\hline Intercept & $-4.513(1.263)$ & $<0.001$ & & \\
Attendance rate & $2.483(0.685)$ & $<0.001$ & 11.98 & $3.13,45.84$ \\
Clinic visits & $-0.523(0.177)$ & 0.003 & 0.59 & $0.42,0.84$ \\
Gender (male) & $0.746(0.307)$ & 0.015 & 2.11 & $1.16,3.85$ \\
Current age & $0.080(0.042)$ & 0.056 & 1.08 & $1.0,1.18$ \\
Diagnosis age & $0.055(0.032)$ & 0.090 & 1.06 & $0.99,1.13$ \\
\hline
\end{tabular}

All significant variables from the univariate analyses were included in the logistic regression model (see Tables 1 and 2): gender, current age, age at diagnosis of diabetes, attendance rate, and number of clinic visits. $\beta$, variable coefficient; SE, standard error; OR, odds ratio; $\mathrm{CI}$, confidence interval.

more likely to decide to remain under our care, while those less motivated are more apt to leave our facility.

Previous studies have reported that metabolic control and diabetes-related outcomes were associated with clinic attendance: patients having frequent contact with the diabetes clinic received continued diabetes education and had a better course and outcome than those with poor or sporadic attendance $[9,12,13]$. Dyer et al. reported that nonattendance was a significant problem in young adult T1D patients; still, glycemic control was significantly improved in those who attended at least three-quarters of their appointments [11]. Interestingly, we found that in young adulthood higher clinic attendance rate was associated with better glycemic control. Thus, attendance rate can serve as a marker of compliance with treatment suggesting that well-controlled patients with a high level of diabetes competency could be scheduled for fewer clinic visits.

The metabolic control of our emerging adult cohort during the ongoing stable phase of diabetes management, as expressed by the median HbAlc of $7.5 \%$, was near the recommended target and $28 \%$ attained the treatment target of 7\% (ADA, ISPAD guidelines). Our findings are in agreement with reports based on individuals with type 1 diabetes participating in the T1D Exchange Clinic Registry and international diabetes registries [14-25]. The incidence of severe hypoglycemia, DKA episodes, and diabetes-related hospitalizations was lower than that reported in other population-based studies [14-25]. These findings are consistent with those of our previous report of a significantly lower percentage of acute diabetes-related complications in young adulthood [26], although it must be noted that the frequencies of severe hypoglycemia, DKA, and hospitalizations may be underreported.

Our data demonstrated that emerging adult males had a better likelihood to attain metabolic treatment targets than females. Gender differences in young adults with type 1 diabetes have been previously investigated [14-17]. In line with previous reports from large databases, $\mathrm{HbAlc}$ levels were consistently lower in males than in females and a higher percentage of male subjects attained treatment targets [14-17].

The employment status of our young adult patients did not influence adherence to scheduled appointments. This is in line with the study by Dyer et al., which found that attendance rates were not associated with employment status [11]. In Israel, patients do not pay out of pocket for clinic visits and paid absence from work for medical reasons is justified. Therefore, not attending scheduled appointments cannot be attributed to the financial burden of the disease. In addition, the geographic distance from the clinic had no impact on clinic attendance. It should be noted that although T1D patients are referred to our tertiary care center from all over the country, most of our population of emerging adults reside in close proximity to the clinic $(\sim 20 \mathrm{~km})$. Moreover, in general most of our health care services are quite easily available, which may not be possible in other countries with a wider geographical population distribution. 
The major strength of this study is the large cohort of T1D patients receiving a similar standard of clinical care and uniform $\mathrm{HbAlc}$ determination in a single tertiary care center. It does, however, have several limitations. The major limitation is that this is a single center clinical observational study in the unique context of continued diabetes care and surveillance of patients until age 30 years of age. Due to its retrospective nature, we could not use questionnaires pertaining to diabetes competency levels and adherence to the diabetes regimen. The reasons for canceling scheduled appointments are not routinely documented in the patient's file. Moreover, the fact that our multidisciplinary team is available 24 hours a day for consultation could have had an impact on glycemic control and may also have resulted in lower attendance. Although our tertiary care center serves all sectors of the Israeli population, including patients of various ethnic origins and social-economic status from both urban and rural areas, our findings may not be applicable for other clinics in Israel or abroad.

In conclusion, our findings suggest that in emerging adult patients with T1D during the ongoing stable phase of diabetes management, a higher attendance rate, rather than the absolute number of clinic visits, was associated with the attainment of better glycemic control. Our institutional policy endorses quarterly visits in order to obtain maximum benefit from the multidisciplinary team of specialists, thus reinforcing good self-care practice. Still, there may be a select group of patients able to maintain good metabolic control with less frequent visits.

\section{Data Availability}

The data used to support the findings of this study are available from the corresponding author upon request.

\section{Disclosure}

This work was performed by Eldad Fisher, M.D., in partial fulfillment of the M.D. thesis requirements of the Sackler Faculty of Medicine, Tel Aviv University, Tel Aviv, Israel.

\section{Conflicts of Interest}

Eldad Fisher, Liora Lazar, Shlomit Shalitin, Michal Yackobovitch-Gavan, Liat de Vries, Tal Oron, Ariel Tenenbaum, Moshe Phillip, and Yael Lebenthal declare that they have no conflict of interest relevant to this article.

\section{Authors' Contributions}

Eldad Fisher made substantial contributions to the conception and design of the work, acquisition, analysis, and interpretation of data for the work, and drafting of the work. Liora Lazar made substantial contributions to the conception of the work, interpretation of data for the work, drafting of the work, and critical revision for important intellectual content. Shlomit Shalitin made substantial contributions to the conception of the work, the interpretation of data for the work, and critical revision for important intellectual content.
Michal Yackobovitch-Gavan made substantial contributions to the design of the work, analysis and interpretation of data for the work, and critical revision for important intellectual content. Liat de Vries, Tal Oron, Ariel Tenenbaum, and Moshe Phillip made substantial contributions to the interpretation of data for the work and critical revision for important intellectual content. Yael Lebenthal made substantial contributions to the conception and design of the work, the interpretation of data for the work, drafting of the work, and critical revision for important intellectual content. All authors gave final approval to the version to be published and agreed to be accountable for all aspects of the work in ensuring that questions related to the accuracy or integrity of any part of the work are appropriately investigated and resolved.

\section{Acknowledgments}

This was an investigator-initiated study supported by a research grant from Novo Nordisk. The authors wish to thank Ruth Fradkin for her editorial assistance.

\section{References}

[1] J. M. Lachin, S. Genuth, D. M. Nathan, B. Zinman, B. N. Rutledge, and for the DCCT/EDIC Research Group, "Effect of glycemic exposure on the risk of microvascular complications in the diabetes control and complications trial-revisited," Diabetes, vol. 57, no. 4, pp. 995-1001, 2008.

[2] H. Keen, "The Diabetes Control and Complications Trial (DCCT)," Health Trends, vol. 26, no. 2, pp. 41-43, 1994.

[3] S. N. Mehta and J. I. Wolfsdorf, "Contemporary management of patients with type 1 diabetes," Endocrinology and Metabolism Clinics of North America, vol. 39, no. 3, pp. 573-593, 2010.

[4] J. J. Arnett, "Emerging adulthood: a theory of development from the late teens through the twenties," The American Psychologist, vol. 55, no. 5, pp. 469-480, 2000.

[5] A. Peters, L. Laffel, and the American Diabetes Association Transitions Working Group, "Diabetes care for emerging adults: recommendations for transition from pediatric to adult diabetes care systems: a position statement of the American Diabetes Association, with representation by the American College of Osteopathic Family Physicians, the American Academy of Pediatrics, the American Association of Clinical Endocrinologists, the American Osteopathic Association, the Centers for Disease Control and Prevention, Children with Diabetes, The Endocrine Society, the International Society for Pediatric and Adolescent Diabetes, Juvenile Diabetes Research Foundation International, the National Diabetes Education Program, and the Pediatric Endocrine Society (formerly Lawson Wilkins Pediatric Endocrine Society)," Diabetes Care, vol. 34, no. 11, pp. 2477-2485, 2011.

[6] F. P. Busse, P. Hiermann, A. Galler et al., "Evaluation of patients' opinion and metabolic control after transfer of young adults with type 1 diabetes from a pediatric diabetes clinic to adult care," Hormone Research, vol. 67, no. 3, pp. 132-138, 2007.

[7] D. Allen and J. Gregory, "The transition from children's to adult diabetes services: understanding the "problem", Diabetic Medicine, vol. 26, no. 2, pp. 162-166, 2009. 
[8] D. S. Lotstein, M. Seid, G. Klingensmith et al., "Transition from pediatric to adult care for youth diagnosed with type 1 diabetes in adolescence," Pediatrics, vol. 131, no. 4, pp. e1062-e1070, 2013.

[9] A. M. Sheehan, A. E. While, and I. Coyne, "The experiences and impact of transition from child to adult healthcare services for young people with type 1 diabetes: a systematic review," Diabetic Medicine, vol. 32, no. 4, pp. 440-458, 2015.

[10] American Diabetes Association, “5. Glycemic targets," Diabetes Care, vol. 39, Supplement 1, pp. S39-S46, 2016.

[11] P. H. Dyer, C. E. Lloyd, R. J. Lancashire, S. C. Bain, and A. H. Barnett, "Factors associated with clinic non-attendance in adults with type 1 diabetes mellitus," Diabetic Medicine, vol. 15, no. 4, pp. 339-343, 1998.

[12] S. K. Lyons, D. J. Becker, and V. S. Helgeson, "Transfer from pediatric to adult health care. Effects on diabetes outcomes," Pediatric Diabetes, vol. 15, no. 1, pp. 10-17, 2014.

[13] Y. Levy-Shraga, N. Elisha, M. Ben-Ami et al., "Glycemic control and clinic attendance of emerging adults with type 1 diabetes at a transition care clinic," Acta Diabetologica, vol. 53, no. 1, pp. 27-33, 2016.

[14] V. Manicardi, G. Russo, A. Napoli et al., "Gender-disparities in adults with type 1 diabetes: more than a quality of care issue. A cross-sectional observational study from the AMD Annals Initiative," PLoS One, vol. 11, no. 10, p. e0162960, 2016.

[15] K. O. Schwab, J. Doerfer, W. Hecker et al., "Spectrum and prevalence of atherogenic risk factors in 27,358 children, adolescents, and young adults with type 1 diabetes: cross-sectional data from the German diabetes documentation and quality management system (DPV)," Diabetes Care, vol. 29, no. 2, pp. 218-225, 2006.

[16] J. A. McKnight, S. H. Wild, M. J. E. Lamb et al., "Glycaemic control of type 1 diabetes in clinical practice early in the 21 st century: an international comparison," Diabetic Medicine, vol. 32, no. 8, pp. 1036-1050, 2015.

[17] V. Zoffmann, D. Vistisen, and M. Due-Christensen, "A crosssectional study of glycaemic control, complications and psychosocial functioning among 18- to 35-year-old adults with type 1 diabetes," Diabetic Medicine, vol. 31, no. 4, pp. 493499, 2014.

[18] K. M. Miller, N. C. Foster, R. W. Beck et al., "Current state of type 1 diabetes treatment in the U.S.: updated data from the T1D Exchange clinic registry," Diabetes Care, vol. 38, no. 6, pp. 971-978, 2015.

[19] G. P. Leese, J. Wang, J. Broomhall et al., "Frequency of severe hypoglycemia requiring emergency treatment in type 1 and type 2 diabetes: a population-based study of health service resource use," Diabetes Care, vol. 26, no. 4, pp. 1176-1180, 2003.

[20] K. Khunti, S. Alsifri, R. Aronson et al., "Impact of hypoglycaemia on patient-reported outcomes from a global, 24-country study of 27,585 people with type 1 and insulin-treated type 2 diabetes," Diabetes Research and Clinical Practice, vol. 130, pp. 121-129, 2017.

[21] C. Sparud-Lundin, I. Öhrn, E. Danielson, and G. Forsander, "Glycaemic control and diabetes care utilization in young adults with type 1 diabetes," Diabetic Medicine, vol. 25, no. 8 , pp. 968-973, 2008.

[22] B. Karges, J. Rosenbauer, T. Kapellen et al., "Hemoglobin A1c levels and risk of severe hypoglycemia in children and young adults with type 1 diabetes from Germany and Austria: a trend analysis in a cohort of 37,539 patients between 1995 and 2012," PLoS Medicine, vol. 11, no. 10, article e1001742, 2014.

[23] B. H. Rowe, M. Singh, C. Villa-Roel et al., “Acute management and outcomes of patients with diabetes mellitus presenting to Canadian emergency departments with hypoglycemia," Canadian Journal of Diabetes, vol. 39, no. 1, pp. 55-64, 2015.

[24] J. Li, D. Yang, J. Yan et al., "Secondary diabetic ketoacidosis and severe hypoglycaemia in patients with established type 1 diabetes mellitus in China: a multicentre registration study," Diabetes/Metabolism Research and Reviews, vol. 30, no. 6, pp. 497-504, 2014.

[25] S. Carlsen, T. Skrivarhaug, G. Thue et al., "Glycemic control and complications in patients with type 1 diabetes-a registry-based longitudinal study of adolescents and young adults," Pediatric Diabetes, vol. 18, no. 3, pp. 188-195, 2017.

[26] Y. Lebenthal, S. Shalitin, M. Yackobovitch-Gavan, M. Phillip, and L. Lazar, "Retrospective comparative analysis of metabolic control and early complications in familial and sporadic type 1 diabetes patients," Journal of Diabetes and its Complications, vol. 26, no. 3, pp. 219-224, 2012. 


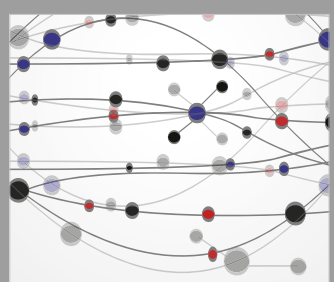

The Scientific World Journal
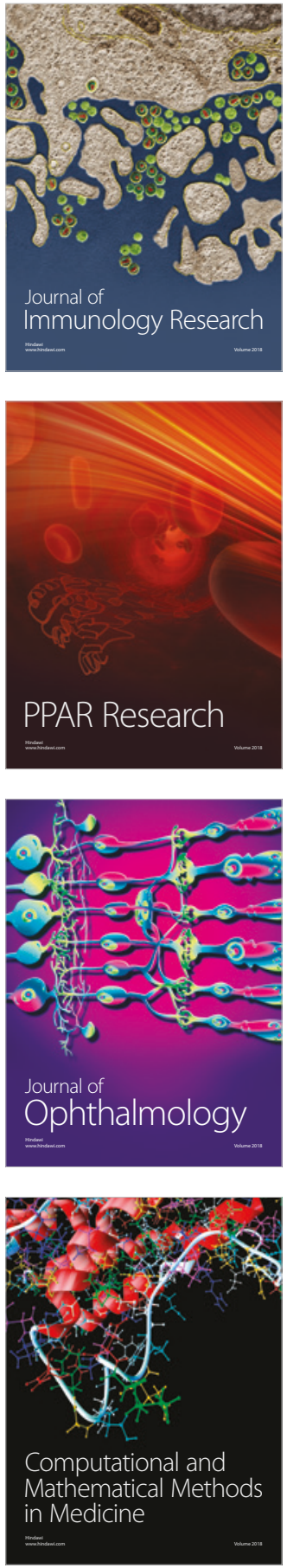

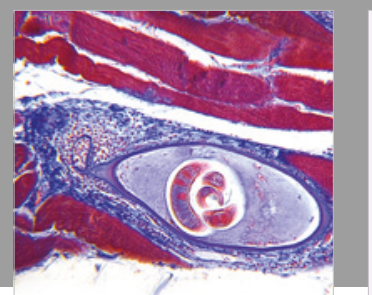

Gastroenterology Research and Practice

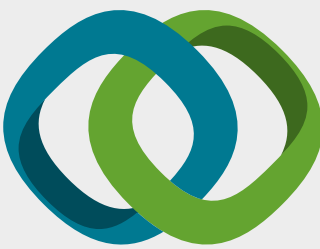

\section{Hindawi}

Submit your manuscripts at

www.hindawi.com
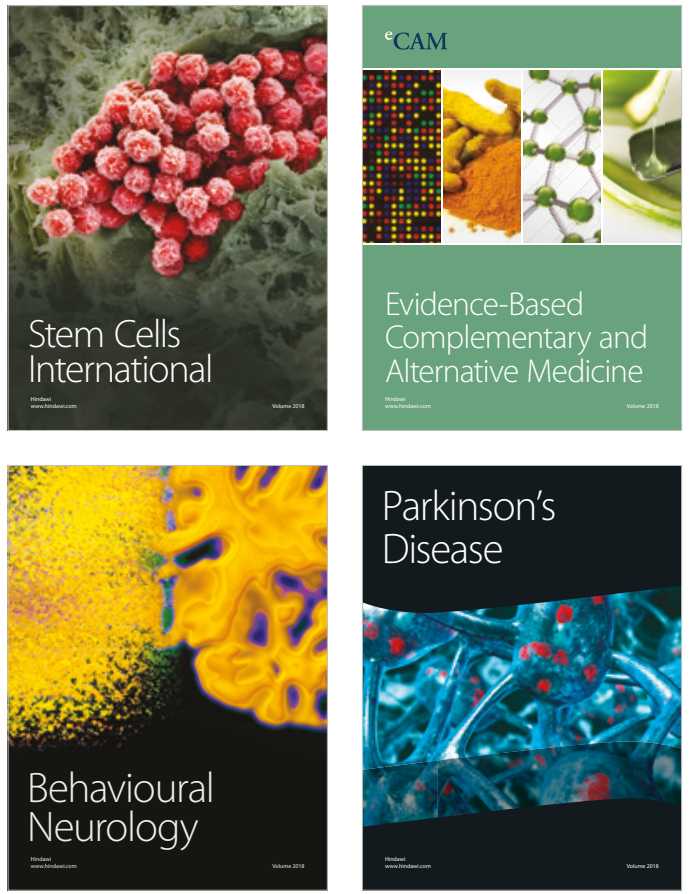



ournal of

Diabetes Research

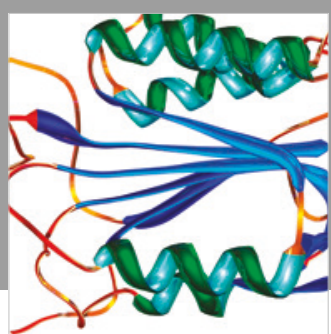

Disease Markers
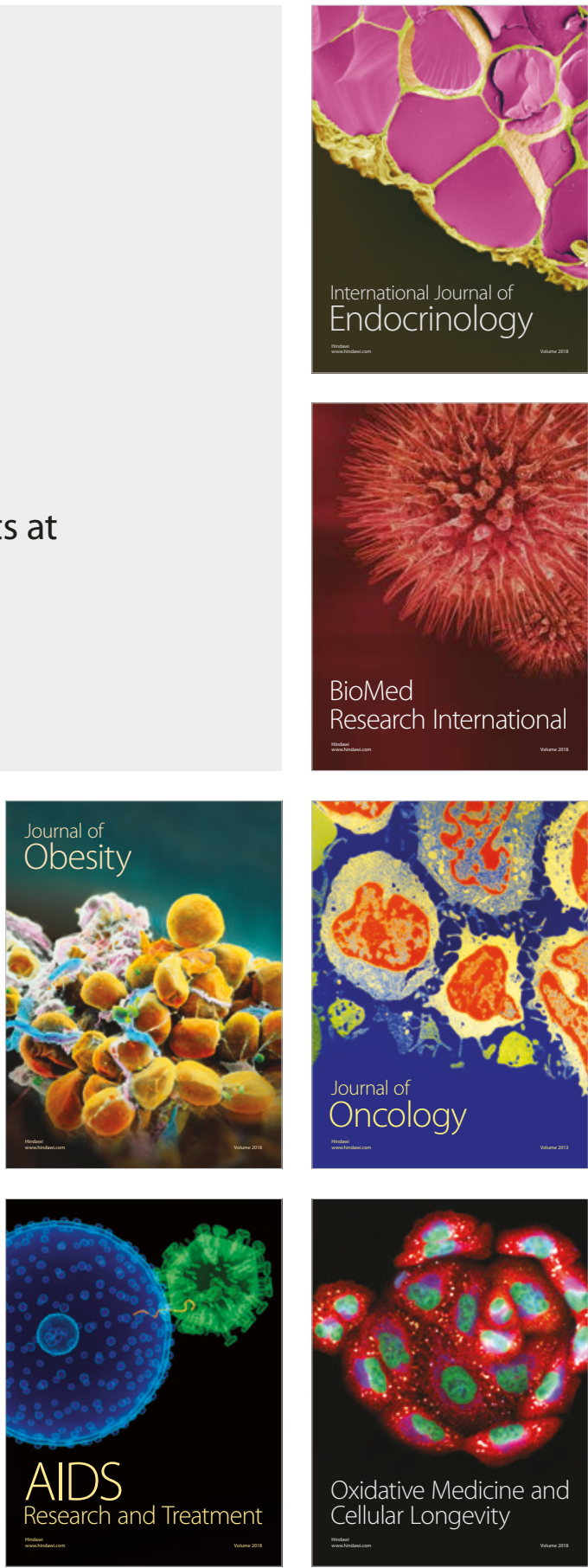